Revista de Psicología Vol. 31 (1), 2013 (ISSN 0254-9247)

\title{
Percepción de anomia, confianza y bienestar: la mirada desde la psicología social $^{1}$
}

\author{
Marcela Muratori $^{2}$, Gisela I. Delfino ${ }^{3} \&$ Elena M. Zubieta ${ }^{4}$ \\ CONICET, UBA
}

La presente investigación busca conocer la percepción de frustración anómica, confianza institucional y bienestar social de los sujetos así como la relación entre estos indicadores. El estudio es descriptivo correlacional, de diseño no experimental transversal, con una muestra no probabilística intencional compuesta por 585 participantes $(34.7 \%$ hombres y $65.3 \%$ mujeres. Edad: $M=28.50 ; S D=11.02$ ) de Buenos Aires y otras ciudades de Argentina. Los resultados arrojan una percepción positiva en el bienestar social con un déficit en la confianza en los otros, bajos niveles de confianza en las instituciones y niveles altos de frustración anómica. Se corrobora la validez convergente y surgen diferencias a partir de variables de agrupación.

Palabras clave: anomia, alienación, confianza (comportamiento social), bienestar, valores sociales.

\begin{abstract}
Anomia Perception, Trust and Well Being: a view from the Social Psychology
An empirical research was carried out with the aim of exploring in levels of anomy, social well-being and institutional trust and their relationship. Is a descriptive correlation study with a non experimental design based on a convenience sample composed by 585 participants $(34.7 \%$ males and $65.3 \%$ females, age: $M=28.50$; $S D=11.02)$ from Buenos Aires and other Argentinian cities. Results show a general positive perception of social well being -with a lack in others confidence-, low levels of institutional trust and high punctuations in anomic frustration. Convergent validity was corroborated and group differences showed up. Key words: anomie, alienation, trust (social behavior), well being, social values.
\end{abstract}



El presente estudio trata de una aproximación a la problemática del bienestar social desde su cercanía con los conceptos de alienación y anomia. Se explora en una perspectiva del bienestar que hace énfasis en la interacción de los individuos con su entorno y en la percepción sobre la capacidad de este para proveerles las condiciones para realizarse como personas. En el área de la psicología social se observa con beneplácito desde hace ya unos ańos una vuelta al interés por estudios abocados a explorar en los aspectos psicosociales de la alienación y la anomia. Tendencia esta que se detecta también en el contexto argentino donde los estudios revelan cierto malestar en los individuos respecto de la capacidad de la sociedad para darles sentido de confianza, de pertenencia y de un propósito común (Benbenaste, Zubieta \& Delfino, 2005; Carballo, 2005; Delfino, 2009; Inglehart, Basáńez, Díez-Medrano, Halman \& Luijkx, 2004). Alienación y anomia son conceptos de origen sociológico que dan cuenta de la integración negativa de los individuos al mundo social en el que se desenvuelven. La alienación tiene como uno de sus componentes a la anomia y ambas nociones describen diferentes

1 Este estudio forma parte de un proyecto financiado por la Secretaría de Ciencia y Técnica de la Universidad de Buenos Aires, Argentina (2008-2010: UBACyT P057; 2011-2014: UBACyT P388).

2 Licenciada en Psicología, Universidad Católica Argentina. Becaria Doctoral Tipo I, CONICET. Ayudante de Trabajos Prácticos e Interina de la Facultad de Psicología de la Universidad de Buenos Aires. Dirección postal: Monseñor Larumbe 855, Martínez (CP 1640). Argentina. Correo electrónico: marcelamuratori@hotmail.com / mmuratori@conicet.gov.ar

3 Licenciada en Psicología, Universidad de Buenos Aires. Doctora en Psicología, Universidad de Buenos Aires. Profesora adjunta interina de la Facultad de Psicología de la Universidad de Buenos Aires. Investigadora asistente CONICET. Dirección postal: Donato Álvarez 86, 6to A. Ciudad Autónoma de Buenos Aires (C1406BNN). Argentina. Correo electrónico: giseladelfino@conicet.gov.ar

4 Licenciada en Sociología, Universidad de Buenos Aires. Doctora en Psicología, Universidad del País Vasco. Profesora adjunta regular Facultad de Psicología, Universidad de Buenos Aires. Investigadora adjunta CONICET. Dirección postal: Tucumán 2162, 8vo. A. Ciudad Autónoma de Buenos Aires (C1050AAR). Argentina. Correo electrónico: elenazubieta@ hotmail.com 
aspectos de la percepción subjetiva del medio ambiente social (Aceituno et al., 2009).

El término alienación está íntimamente relacionado al análisis de Marx (1844/1985) acerca de la enajenación que el trabajo provoca en las relaciones capitalistas de producción pero, de manera más general, refiere a un estado o proceso en los cuales la persona pierde o queda extrañada respecto de algo que originalmente poseía. Fue Hegel (1807/1971), quien desde la metafísica, relacionó la alienación o enajenación a la conciencia infeliz que surge cuando esta se experimenta separada de la realidad a la cual pertenece provocando sentimientos de desgarramiento, desunión y desposeimiento. Cuando se habla de alienación se alude a aquello que vuelve al individuo contra su esencia, que en vez de liberarlo lo cosifica. Múltiples disciplinas han recuperado el concepto de alienación generando importante cantidad de definiciones y abordajes e instalando al mismo tiempo la discusión acerca de su condición objetiva, subjetiva o psicológica. La mirada desde la psicología social no implica optar por una u otra condición sino considerarlo como resultado sintético de la interacción individuo-grupo. En esta perspectiva, la alienación puede tomar la forma de los procesos de despersonalización o des individuación y a su vez, como señalan Grossi y Ovejero (1994), variar o ser específicos a ámbitos como la familia, el trabajo, la religión, los amigos o la política.

Sabucedo (1988) detalla los aspectos subjetivos de la alienación que propone Seeman (1982) y que estarían asociados a cambios en las estructuras sociales así como a consecuencias psicosociales tales como el desorden mental, la pasividad política o la aversión al trabajo, entre otros. Estos cinco indicadores con:

1. Impotencia o falta de poder — powerlessness — : la expectativa o probabilidad mantenida por el individuo de que su propia conducta no puede determinar la ocurrencia de resultados o refuerzos que él desea. Ya sea que se trate de situaciones complejas y cambiantes, o en situaciones laborales alienantes, las personas perciben que carecen de control sobre los sucesos vitales o sobre su trabajo. 
2. Falta de sentido — meaninglessness-: refiere a la incomprensión del sentido de los actos y acontecimientos que involucran al sujeto. Se produce cuando el desarrollo social genera situaciones en las que los individuos sienten que la existencia carece de significado. Los hechos sociales se vuelven incomprensibles e inexplicables.

3. Anomia o falta de normas — normlessness - : una expectativa alta de que se requieren conductas o medios no aprobados para lograr determinadas metas. Es el rechazo de la comunidad como fuente de normas.

4. Aislamiento social o falta de integración —-isolation-: supone un sentimiento de soledad y abandono. Refiere a situaciones en las que se concede poco valor a metas o creencias consideradas centrales y evaluadas positivamente en una sociedad. La soledad se asocia no solo a la baja frecuencia objetiva de contacto sino también a un contacto social subjetivamente insatisfactorio.

5. Autoenajenación o autoextrańamiento —self-estrangement-: implica sentimientos por parte de los individuos de que se es menos de lo que uno podría realmente ser si las circunstancias de la sociedad fueran otras. Refiere a la diferencia entre lo que se quiere ser y lo que se es realmente. Es necesario destacar la diferencia entre el desprecio del yo, asociado a una autoestima baja, o la participación en actividades laborales alienadas en las que no hay provecho intrínseco para las personas, de la falsa conciencia o creencia en ideologías que justifican el sistema social, ocultando los intereses reales de los grupos.

En lo que hace al concepto de anomia, este se asocia al sociólogo Emile Durkheim y a su interés por reflejar las tensiones entre individuo y sociedad. En "La división del trabajo social" (1893/1973) Durkheim define a la anomia como un problema moral producto del deterioro o ruptura de los lazos sociales y el decaimiento de la solidaridad. La asocia también con la transformación de las representaciones colectivas en términos de la regulación de expectativas y deseos. En "El suicidio" (1897/1976) complementa el concepto indicando que las 
sociedades regulan e imponen límites a las necesidades y deseos de los individuos. Desde la sociología, Merton (1938) relaciona a la anomia con el problema de la disponibilidad de medios institucionales para alcanzar metas definidas culturalmente. Por su parte, las interpretaciones psicológicas asocian a la anomia con sentimientos de impotencia, desamparo y desesperanza (Parales-Quenza, 2008). En lo que hace a la psicología social, la percepción de anomia deviene del desequilibrio entre las metas que una sociedad se propone y propone a los individuos y los logros reales de estos, y de su efecto en sus expectativas y propósitos. Mclver (1950 en López Fernández, 2009) la define como un estado de ánimo en el que el sentido de cohesión social del sujeto está fragmentado o debilitado. El sujeto deja de preocuparse por el entorno y actúa a partir de sus propios impulsos; se centra en su persona ya que las reglas y fines sociales, sin continuidad ni sentido de obligación, no tienen valor en su vida diaria.

Srole (1956) profundiza el análisis de la anomia como noción psicosocial enfatizando el estudio de los sentimientos y percepciones individuales. El autor plantea una percepción de la sociedad, y una autopercepción, desintegrada y una falta de involucramiento de los individuos con su entorno. Establece un conjunto de indicadores a fin de medir el grado de anomia que los miembros sienten con respecto a la sociedad, siendo uno de los extremos la relación adecuada con los otros, y el otro, la alienación. El hecho de que un sujeto se sitúe en uno u otro extremo se considera indicador de fenómenos tales como la percepción de los líderes frente a las necesidades de los miembros de la comunidad, la percepción de insatisfacción y de desorganización respecto a la sociedad, la percepción sobre la capacidad para cumplir metas individuales, la sensación de nimiedad, y el sentimiento de compañerismo entre los sujetos de una sociedad. A medida que la persona vivencia una mayor insatisfacción y desorganización se vuelve más anómico. Desde la perspectiva eudaimónica del bienestar, el bienestar social es considerado como la contra cara de la alienación social (Basabe \& Páez, 2006) ya que Keyes (1998) lo define como la valoración que las personas hacen de sus circunstancias y del funcionamiento de la sociedad. El autor 
distingue cinco dimensiones que conforman el bienestar social: (1) Integración social, entendida como la evaluación de la calidad de las relaciones que los miembros mantienen con la sociedad y la comunidad, implica estar y sentirse perteneciente a un grupo; (2) Aceptación social, referida no solo a la aceptación de los aspectos positivos y negativos de nuestra propia vida, sino también a la confianza, aprobación y actitudes positivas hacia los otros; (3) Contribución social, comprendida como el sentimiento de utilidad, implica el hecho de sentir que uno puede aportar algo valioso al bien común; (4) Actualización social, es la confianza en el futuro de la sociedad, confianza en el progreso y en cambio social, en el potencial de crecimiento y desarrollo, en su capacidad para producir bienestar; y (5) Coherencia social, que tiene que ver con la capacidad para comprender la dinámica de la sociedad, de percibir la cualidad, organización y funcionamiento del mundo social, hallando una lógica en los acontecimientos que rodean.

Estas cinco dimensiones planteadas por Keyes (1998) representan la versión positiva de las facetas de las dimensiones subjetivas que Seeman (1991) destaca respecto de la alienación. A saber: (a) el aislamiento social en la alienación, que refiere al bajo apoyo social objetivo y subjetivo, es el equivalente en el bienestar social a una baja integración social, a no sentirse parte de la comunidad, a no experimentar apoyo ni que se comparten cosas en común con el colectivo; (b) el auto-extrańamiento personal, en tanto que baja auto-estima y desconfianza en el otro, corresponde a la dimensión de aceptación del bienestar en el que la persona exhibe una actitud positiva hacia los otros en general aún cuando por momentos la conducta sea compleja o incomprensible; (c) la ausencia de sentido y la falta de normas, anomia, en la alienación equivalen en el bienestar social a la baja contribución social. Se caracteriza por la percepción de los individuos de ausencia de sentido o de propósito en la vida y de un pobre sentimiento de tener algo positivo que dar a la sociedad y de que sus actividades sean valoradas; (d) tanto el auto-extrańamiento en relación a la actividad, cuando el trabajo provee poca satisfacción intrínseca, como la creencia que el sujeto no está implicado en sus actividades —baja implicación en el trabajo_--, es el correlato de baja actualización 
social o creer que el mundo social se desarrolla o puede desarrollarse para mejor; y (e) la falta de poder en la alienación equivale a una baja coherencia en el bienestar social, es decir, la baja expectativa de control de lo que ocurre o bajo dominio del entorno inmediato, así como el bajo control del mundo social. Baja presencia de la creencia que el mundo es predecible, inteligible y lógico, y por ende controlable, así como poca preocupación e interés en la comunidad.

Junto al bienestar social, otro elemento crítico asociado a estados de alienación y anomia es la confianza o desconfianza en las instituciones, aspecto este profundizado sobre todo por la psicología política que específicamente se ha interesado por la alienación política. Grossi y Ovejero (1994) sostienen que junto a la impotencia o falta de poder, la desconfianza política es uno de los indicadores más relevantes en la actualidad para evaluar la alienación política. Esta se refiere a una valoración negativa de las instituciones y su funcionamiento, al sentimiento de que el gobierno y los políticos quienes lo ejecutan son deshonestos, corruptos y no creíbles. Long (1990) define a la alienación política como "una respuesta afectiva por parte del individuo al sistema político y a sus líderes, que es básicamente de naturaleza negativa” (p. 88) y que se caracteriza por cuatro sentimientos interrelacionados: (a) ineficacia política, (b) descontento en relación con los resultados del sistema político, (c) desconfianza hacia los motivos y comportamientos de los líderes políticos, y (d) un sentimiento de alejamiento del sistema político. Distintos estudios, entre ellos uno realizado en el contexto argentino, muestran cómo la alienación política lleva a los individuos a la inactividad, a rechazar las formas convencionales de participación y a preferir formas no convencionales de participación social (Delfino, 2009; Herring, 1989).

Inglehart et al. (2004) sostienen que la falta de confianza en las instituciones y en las relaciones con otros, y el no respetar las normas conducen a una falta de consenso sobre las conductas sociales deseables y facilitan la conducta desviada. Para Rodríguez Kauth (2001), la falta de confianza aumenta el sentimiento de impotencia y desesperanza favoreciendo las condiciones para el resquebrajamiento del entramado social que se refleja en la pérdida de la solidaridad social y 
el aumento del individualismo egoísta. Benbenaste, Etchezahar y Del Río (2008) destacan que el nivel y tipo de comportamiento anómico en una población no solo afectan la calidad de vida y las relaciones sociales sino que también inciden en las actitudes, y por ende, en la confianza hacia las instituciones. En el marco de la problemática latinoamericana, Parales-Quenza (2008) señala que la anomia ha sido considerada principalmente en relación a la ilegalidad, corrupción e inobservancia de normas y reglas, adquiriendo una significación particular. En la región, la anomia emergería como producto de proyectos nacionales incompletos y excluyentes.

Como se indicara al inicio, diversos estudios recientes realizados en el contexto argentino (Benbenaste et al., 2005; Carballo, 2005; Delfino, 2009) dan cuenta de niveles muy bajos de confianza en las instituciones, especialmente en lo que hace a los partidos políticos y a los poderes legislativo y ejecutivo, reflejando la crisis de legitimidad que atraviesan actualmente las instituciones políticas (Pegoraro, 2000). En este marco es que se continúa una línea de indagación psicosocial acerca del vínculo que los individuos establecen con el entorno, de su confianza en el futuro de la sociedad y de la capacidad de esta para proveerles bienestar. Se realizó entonces un estudio empírico con el objetivo de conocer los niveles de frustración anómica, confianza institucional y bienestar social percibidos por los sujetos así como la relación entre los distintos indicadores.

\section{Método}

Se trata de un estudio descriptivo, correlacional, de diseño no experimental transversal.

\section{Participantes}

La muestra, no probabilística intencional, está compuesta por 585 personas (34.7\% hombres y $65.3 \%$ mujeres) con una media de edad de 
$28.50(S D=11.02 ; M d n=25 ;$ rango $=17-66)$. El $64.3 \%$ es soltero, el $30.2 \%$ casado o convive con su pareja y un $5 \%$ separado o divorciado. El 23.8\% tiene hijos.

En relación al lugar de residencia, el $32.8 \%$ habita en la Ciudad Autónoma de Buenos Aires, el 35.2\% en el conurbano bonaerense y el 29.7\% en el Interior del país.

Del total de la muestra, el $33.5 \%$ solo estudia, el $31.3 \%$ solo trabaja, el $33.8 \%$ estudia y trabaja y el $1.4 \%$ no estudia ni trabaja.

\section{Medidas e instrumentos de medida}

El cuestionario utilizado es de formato autoadministrado y estuvo integrado por una sección de datos personales, evaluada al final del mismo, y por las siguientes escalas:

1. Frustración Anómica de Srole y Christie (Basabe \& Páez, 2006): evalúa la percepción de un estado social negativo para la persona, que la aliena o no responde a sus necesidades, focalizando en el componente de anomia o falta de normas que le den sentido y orienten la conducta social. Incluye también preguntas que refieren a la alienación como componente de la anomia (por ejemplo: La mayoría de los que mandan se interesan mucho por los problemas del hombre de la calle; No hay maneras buenas o malas de hacer dinero, sino manera fáciles o difíciles). Consta de 10 ítems con opción de respuesta dicotómica $(0=$ no, $1=$ si), con una fiabilidad de $\alpha=0.44$, índice aceptable de acuerdo a lo señalado por Basabe \& Páez (2006) para estudios anteriores sobre esta misma escala.

2. Bienestar Social de Keyes (Blanco \& Díaz, 2005): expone criterios públicos e interpersonales de evaluación de un buen funcionamiento psicológico. Está compuesta por 33 ítems en forma de afirmaciones como: Siento que soy una parte importante de mi comunidad; Creo que no se debe confiar en la gente; Creo que puedo aportar algo al mundo; Para mi el progreso social es algo que no existe; No entiendo lo que está pasando en el mundo, con un continuo de respuesta de 1 (totalmente en desacuerdo) a 5 (totalmente de acuerdo). Evalúa la percepción que las 
personas tienen sobre cinco aspectos del entorno social que facilitan su bienestar: integración social (7 ítems, $\alpha=0.72$ ), aceptación social (8 ítems, $\alpha=0.84$ ), contribución social (6 ítems, $\alpha=0.79$ ), actualización social (6 ítems, $\alpha=0.67$ ) y coherencia social (6 ítems, $\alpha=0.54$ ).

3. Confianza en las Instituciones (Inglehart et al., 2004): indica el nivel general de confianza que los individuos manifiestan respecto de ocho instituciones sociales (tales como: Policía, Partidos Políticos, Iglesia Católica, entre otras) en un continuo de respuesta de 1 (no confía) a 4 (confía mucho) ( $\alpha=0.70)$. Valores medios por encima de 2.5 indican confianza institucional, tanto en el promedio de las ocho instituciones como en las puntuaciones específicas de cada una de ellas.

\section{Procedimiento}

La administración del cuestionario se realizó de forma individual y los sujetos fueron invitados a participar voluntariamente y de forma anónima, según las normas y estándares para la investigación con seres humanos.

\section{Resultados}

Como puede observarse en la Tabla 1, los datos muestran un buen nivel en general en lo que hace a los criterios sociales de bienestar. Cuatro de los cinco indicadores propuestos por Keyes (1998) resultan medio-altos: contribución, integración, coherencia y actualización, siendo la puntuación media de aceptación social algo baja, lo que refleja una falta de confianza en los otros y un auto-extrańamiento personal asociado a la baja autoestima. Los participantes se sienten parte de la comunidad, perciben satisfechas sus necesidades de apego, afiliación y pertenencia. Sienten que pueden hacer un aporte positivo y valioso a la sociedad y ver en ella cierta lógica y previsión. Sin embargo, hay un déficit en la confianza interpersonal y en las actitudes positivas hacia los otros. La baja aceptación orienta las tendencias comunales hacia el individualismo. 


\section{Tabla 1}

Medias y desvios estándar en la escala de Bienestar Social ( 1 = totalmente en desacuerdo, 5= totalmente de acuerdo)

\begin{tabular}{lll}
\hline Dimensión & $M$ & $S D$ \\
\hline Contribución social & 3.89 & .71 \\
Integración social & 3.72 & .62 \\
Coherencia social & 3.41 & .62 \\
Actualización social & 3.18 & .66 \\
Aceptación social & 2.95 & .73 \\
\hline
\end{tabular}

Los niveles de frustración anómica son altos, la puntuación media es de $4.55(S D=1.66)$ cuando en general oscilan entre 3 y 4 (Basabe $\&$ Páez, 2006). Esto indica la dificultad de los individuos para percibirse involucrados con su entorno. Alude a los sentimientos individuales de aspectos como la distancia de los líderes respecto de la comunidad, la desorganización de la sociedad y la imposibilidad de cumplir metas individuales y de nimiedad. Esto último es similar a lo que expresa la dimensión de aceptación en el bienestar social y enfatiza también la falta de compañerismo. En palabras de Mclver (1950 en López Fernández, 2009), hay una percepción de fragmentación en los participantes en relación al sentido de cohesión social por lo que mostrarán mayor tendencia a centrarse en su persona al experimentar que muchas de las reglas y valores sociales no tienen valor. La media de confianza en las instituciones es baja (ver Tabla 2). Los Partidos Políticos y el Poder Ejecutivo son las dos instituciones que más desconfianza suscitan, mientras que la Iglesia Católica es la que genera más confianza, seguida de la Policía y las Fuerzas Armadas. El Poder Legislativo, el Poder Judicial y los gremios y sindicatos exhiben bajas puntuaciones. Como se mencionara, la desconfianza en las instituciones es uno de los estados críticos de la alienación y anomia y junto a la falta de poder, o coherencia en el bienestar social, es un factor relevante de la alienación política (Grossi \& Ovejero, 1994). 


\section{Tabla 2}

Puntuaciones medias en el nivel de confianza en las instituciones ( 1 = ninguna confianza, $4=$ much a confianza)

\begin{tabular}{lll}
\hline Instituciones & $M$ & $S D$ \\
\hline Confianza general & 1.83 & .40 \\
\hline Iglesia Católica & 2.02 & .94 \\
Policía & 1.94 & .61 \\
Fuerzas Armadas & 1.93 & .77 \\
Poder Legislativo & 1.87 & .62 \\
Poder Judicial & 1.86 & .68 \\
Gremios / Sindicatos & 1.78 & .74 \\
Poder Ejecutivo & 1.70 & .63 \\
Partidos Políticos & 1.56 & .57 \\
\hline
\end{tabular}

Inglehart et al. (2004) destacan que la falta de confianza en las instituciones y en las relaciones con otros, así como el no respeto a las normas, llevan a una falta de consenso sobre las conductas sociales deseables y facilitan las conductas desviadas. Son muchos los estudios que dan cuenta de la anomia como aquello que se opone al bienestar social (Laca Arocena, Mejía Ceballos \& Yañez Velasco, 2010; Laca Arocena, Santana Aguilar, Ochoa Madrigal \& Mejía Ceballos, 2011). Si bien las puntuaciones en bienestar social de esta muestra no son bajos, aquella relación se corrobora ya que todas las dimensiones de bienestar social se asocian de forma negativa con la frustración anómica (ver Tabla 3). En el mismo sentido, la anomia correlaciona negativa y significativamente con la confianza en las instituciones, es decir, cuanto más deshonestas y poco creíbles se percibe a las instituciones, cuanto más es la desconfianza hacia ellas, mayores son los niveles de frustración anómica, y también de bienestar social. 


\section{Tabla 3}

Relación entre Bienestar Social, Frustración Anómica y Confianza en las Instituciones

\begin{tabular}{lll}
\hline Bienestar Social & Anomia & Confianza \\
\hline Contribución & $-.183^{* *}$ & $.095^{*}$ \\
Integración & $-.284^{* *}$ & $.156^{* *}$ \\
Coherencia & $-.208^{* *}$ & $.116^{* *}$ \\
Actualización & $-.383^{* *}$ & $.304^{* *}$ \\
Aceptación & $-.476^{* *}$ & $.195^{* *}$ \\
\hline Anomia & & $-.282^{* *}$ \\
\hline
\end{tabular}

Nota: los valores presentados son $r$ de Pearson. ${ }^{*} p<.05{ }^{* *} p<.01$

\section{Incidencia de las variables sociodemográficas}

En relación al sexo, se observa que las mujeres, en comparación a los hombres, presentan mayores niveles de integración $(t(583)=-2.03$; $\left.p=.04 ; M_{\text {Mujeres }}=3.76 ; M_{\text {Hombres }}=3.65\right)$, no habiéndose hallado ninguna otra diferencia significativa en relación a las dimensiones restantes del bienestar, la frustración anómica y la confianza institucional. Es decir, las mujeres de esta evalúan mejor que sus pares masculinos las relaciones con la comunidad, sintiendo mayores niveles de pertenencia. Estos datos van en la línea con lo hallado en un estudio previo (Zubieta, Muratori \& Fernández, 2011) en el que se encontró también que las mujeres se perciben más integradas que los hombres a la vez que sienten mayor confianza en el progreso de la sociedad y el cambio. Asimismo, en lo que hace a género y valores, los datos corroboran que las mujeres, además de mayor utilidad, se orientan a valores más expresivos comunales orientados a lo social mientras que los hombres tienden a motivos más instrumentales y centrados en la persona (Zubieta, 2008).

En función a la edad de los participantes, surgen diferencias en la confianza en las instituciones. Los sujetos mayores a 29 ańos puntúan más bajo que quienes tienen menos de 28 años $(F(2.582)=12.37$; $p=.00$. Mayores 29 ańos $=1.73$; entre 23 y 28 ańos $=1.85$; menores a 
22 años = 1.92). A medida que se avanza en la edad se descree más de las instituciones. No surgen diferencias en las dimensiones de bienestar social ni en los niveles de frustración anómica. En términos de valores, la edad se asocia de manera positiva con la conservación, cuanto más años se cumple es mayor el apego a la seguridad y la conformidad y, a la inversa, la juventud se asocia a la estimulación, los desafíos y la búsqueda de independencia. La desconfianza en las instituciones de los participantes de mayor edad en esta muestra puede ser una señal de mayor alienación política en términos de percepción de falta de poder y de baja expectativa de control del entorno que refieren a valores de mayor conservación (Zubieta, 2008).

En lo que hace al estado civil y el bienestar social, los solteros (3.77) se sienten más integrados que quienes estan casados o conviven (3.67), o están divorciados $(3.52)(F(2.579)=3.31 ; p=.04)$. Los divorciados (3.56) por su parte, son quienes perciben mayor coherencia mientras que la mayor diferencia en esta dimensión se da entre solteros (3.45) y casados (3.30). Estos últimos presentan mayores dificultades para comprender la dinámica social o encontrar una lógica en los acontencimientos que los rodean $(F(2.579)=4.31 ; p=.01)$. No hay diferencias en las otras dimensiones de bienestar social ni en frustración anómica, aunque si en la confianza institucional. Son lo solteros (1.89) quienes más confian en las instituciones, seguidos por los casados (1.73) y por último los divorciados $(1.69)(F(2.579)=12.43 ; p=.00)$. En estos datos, se perciben con mejor bienestar quienes explicitan estar solos mostrando una tendencia diferente a la clásica comprobación de Durkheim (1897/1976) acerca de la relación negativa de los casados con el suicidio. Asimismo, recordemos que en el bienestar social se detectaba una orientación a lo personal por sobre lo social por lo que probablemente aquella tendencia se reforzaría con esta relación a favor de quienes están solos y sin responsabilidades más que ellos mismos. Dato que se completa al analizar a los participantes en función de si tienen o no hijos.

En relación al bienestar social, los participantes que son padres perciben menor integración y menor coherencia. Es decir, evalúan de manera más negativa la calidad de las relaciones que mantienen con la 
comunidad y muestran mayor dificultad para comprender los acontecimientos de la dinámica social (Integración: $t(200,696)=-2.29 ; p=.02$. Con hijos=3.61; $\sin$ hijos=3.76. Coherencia: $t(210,158)=-3.21 ; p=.00$. Con hijos=3.26; $\sin$ hijos=3.46). De manera esperable, son también los participantes con hijos quienes exhiben niveles más altos de frustración anómica y confían menos en las instituciones (Anomia: $t(579)=2.16$; $p=.03$; Con hijos=4.83; $\sin$ hijos $=4.48$. Confianza: $t(207,174)=-3.35$; $p=.00$. Con hijos=1.73; sin hijos=1.87). El ser padres se relaciona con mayores niveles de preocupación, de malestar, de problemas para encontrar sentido por parte del entorno y a sus instituciones.

Por último, al agrupar a los participantes en función de la actividad que realizan, quienes estudian y trabajan presentan mayores niveles de aceptación social (03.03), en comparación con aquellos que realizan una sola actividad — solo estudian (2.99) o solo trabajan (2.82) - o ninguna, ni estudian ni trabajan $(2.91)(F(3.581)=2.78 ; p=.00)$. Por lo tanto, el realizar ambas actividades hace que los individuos perciban mayor aceptación y tengan actitudes positivas hacia los otros. Por su parte, los que estudian, ya sea como única actividad (3.49) o acompañada por el trabajo (3.48), perciben mayor coherencia social que quienes solo trabajan (3.27) o no realizan ninguna actividad $(2.96)(F(3.581)=$ $7.03 ; p=.00)$. Los que solo estudian (1.93) confían más en las instituciones que el resto de los participantes: estudia y trabaja (1.79), solo trabaja (1.79), no estudia ni trabaja $(1.52)(F(3.581)=6.78 ; p=.00)$. Este dato está ratificando la relación reportada entre los más jóvenes y la confianza en las instituciones ya que son los de menor edad los que solo estudian. El estudio y trabajo son vehículos de percepción de aceptación mientras que el estudio lo es de la coherencia social, es decir del hecho de comprender la dinámica social, de encontrar una lógica a los acontecimientos. Este hallazgo se refuerza con los niveles de frustración anómica que arroja diferencias entre quienes solo trabajan (4.79) y quienes estudian y trabajan (4.29), siendo los últimos los menos anómicos, seguidos por los que solo estudian (4.55). Por otra parte, los sujetos que no estudian ni trabajan son quienes presentan los niveles más altos de frustración anómica $(5.25)(F(3.581)=3.44 ; p=0.02)$. El hecho de 
realizar una actividad amortigua el sentimiento de desintegración o de baja percepción positiva del entorno y de uno mismo.

\section{Discusión}

Los datos aquí reportados muestran que los participantes perciben en general de manera positiva su relación con el entorno y de lo que este les provee para satisfacer sus necesidades como persona social. Hay sin embargo un déficit en lo que hace a la aceptación social, una baja confianza en las instituciones y niveles altos de frustración anómica. Los datos en relación al bienestar social corroboran lo hallado en un estudio previo (Zubieta \& Delfino, 2010) acerca de la dificultad en los individuos respecto de la aceptación, de lograr confianza y actitudes positivas hacia los otros. Recordemos que la dimensión de aceptación en el bienestar social desarrollado por Keyes (1998) es el correlato al auto-extrańamiento personal planteado por Seeman (1991) como dimensión subjetiva de la alienación. Esta última tiene que ver con una baja auto-estima y con la disonancia de querer tener una actitud positiva hacia los otros que no se traslada a las conductas que aparecen como complejas e incomprensibles, más orientadas hacia la persona y alejadas del compañerismo o la cooperación.

A su vez, se ratifica la tendencia registrada sobre la escasa confianza en las instituciones (Zubieta, Delfino \& Fernández, 2008), asociada a la alienación política y a su dimensión de impotencia o falta de poder en tanto probabilidad percibida por los individuos de que sus conductas determinen los resultados o refuerzos deseados (Seeman, 1971 en Aceituno et al., 2009). Las puntuaciones altas en anomia refuerzan esta tendencia ya que da cuenta de la percepción de cierto estado social negativo en el que no se observan normas claras que rijan y den sentido y orientación a la conducta social. Este descreimiento se relaciona con los hallazgos obtenidos en un estudio sobre la percepción de la norma y el sistema normativo argentino (Beramendi \& Zubieta, 2011) en el que los participantes exhiben la ausencia de un marco normativo de 
contención donde la forma de lograr los objetivos que se promueven socialmente es a través de comportamientos desviados, por medio del uso de conocidos o de entrar en un sistema alternativo de trasgresión de la norma. Las puntuaciones satisfactorias en las dimensiones del bienestar social junto a una baja confianza institucional y una anomia relativamente alta pueden estar relacionadas con lo planteado por Laca Arocena et al. (2010) acerca de que los datos con esta tendencia pueden pensarse en el marco de la cultura de un país donde la anomia sería percibida como una característica estable más que como un síntoma de una época de crisis. Vale recordar aquí lo que Inglehart et al. (2004) señalan acerca de la falta de confianza en las instituciones y en las relaciones con otros que, junto con el no respeto a las normas conducen a una falta de consenso sobre las conductas sociales deseables y facilitan la conducta desviada. Conforme a la hipótesis de validez convergente, las dimensiones del bienestar social correlacionan negativa y significativamente con la anomia, y positiva y significativamente con la confianza en las instituciones. Se refuerza entonces la idea del bienestar social como la cara opuesta de la alienación y la anomia así como de la desconfianza institucional como indicador de alienación política.

Por último, algunas diferencias interesantes surgen al introducir algunas variables de agrupación como el sexo, la edad, el estar en pareja o el hecho de realizar alguna actividad. Las mujeres perciben mejor que los hombres su relación con el entorno en términos de integración y evalúan mejor que sus pares masculinos la calidad de las relaciones con la sociedad. Estos datos van en la línea de exploraciones previas (Zubieta \& Delfino, 2010; Zubieta et al., 2011) en las que, además de una mayor integración, en términos de bienestar psicológico, las mujeres manifestaron experimentar un mayor dominio del entorno y propósito en la vida en comparación con los hombres. Se asociaba los resultados obtenidos a la mejora que las mujeres experimentan en lo que hace a un mayor reconocimiento en cuestiones de la esfera pública que aportan a un buen funcionamiento psicológico relacionado con el locus de control interno, a la alta auto-eficacia y a la motivación para actuar y desarrollarse. Asimismo, en términos de valores se podría 
decir que se traduce en una utilidad social actual que parece pasar por motivaciones comunales expresivas asociadas culturalmente a atributos femeninos. Una nueva competencia social que se refleja también en la estimulación de liderazgos más transformacionales que sumen a lo agéntico instrumental masculino lo expresivo comunal femenino (Zubieta, Beramendi, Sosa \& Torres, 2010).

De manera interesante, el estar soltero o divorciado parece aportar al bienestar en términos de encontrar más lógica a la dinámica social y a confiar más en las instituciones. Exactamente la misma tendencia se observa en relación a tener responsabilidad sobre hijos ya que el ser padres aumenta, probablemente de manera esperable en el contexto actual, la preocupación por una lógica social que se percibe incomprensible y la desconfianza en que las instituciones políticas para revertir la situación. $\mathrm{O}$, como se señalara previamente, la presencia de un contexto en el que la sensación de extrańamiento tiene que ver más con lo personal y con la autoestima y que, de forma paradojal, se resuelve con una progresiva distancia de fines y reglas sociales que se viven como ajenas. Finalmente, el sentirse activo a través del trabajo y/o el estudio, amortiguan la frustración anómica haciendo que los individuos se sientan más integrados y que tengan una relación más satisfactoria con el entorno y con ellos mismos. Esto refiere a la sensación de autoextrańamiento o enajenación con uno mismo, a la diferencia entre lo que se quiere hacer y lo que se hace, reflejado en una baja autoestima y a la participación en actividades laborales alienadas o de escasa o nula motivación intrínseca (Blanco \& Díaz, 2007). La edad aumenta la desconfianza en las instituciones, a la vez que refuerza el hecho de que los jóvenes, que están en su mayoría estudiando, se sienten probablemente más útiles e integrados a través de la actividad que realizan. Por su parte, la mayor edad refleja una mayor conservación que refuerza la seguridad y la conformidad lo que, en términos más políticos, podría asociarse actualmente a una mayor crítica a las instituciones o a una mayor percepción de alienación política.

En síntesis, indagar en las consecuencias psicosociales de estados de alienación o anómicos a partir de los sentimientos y las percepcio- 
nes individuales permite hacer relevante, además de la relación entre constructos cercanos que aportan luz desde distintas aristas, aspectos como la tendencia a distanciarse del grupo o comunidad reforzando lo individual y estimulando la aparición de normas personales por sobre las sociales; la utilidad social entendida de forma disonante en términos comunales-emocionales a la vez que probablemente asociada a la no responsabilidad en términos de pareja e hijos y a la juventud. Por último, el trabajo y el estudio, así como las actividades de ocio, amortiguan la percepción de malestar, frustración y desconfianza.

\section{Referencias}

Aceituno, R., Asún, R., Ruiz, S., Reinoso, A., Venegas, J. I. \& Corbalán, F. (2009). Anomia y alienación en estudiantes secundarios de Santiago de Chile: resultados iniciales de un estudio comparativo 1989-2007. Psykhe, 18(2), 3-18.

Basabe, N. \& Páez, D. (2006). Alienación, anomia y la escala de frustración anómica de Srole y Christie. Documento técnico. Universidad del País Vasco.

Benbenaste, N., Delfino, G. I. \& Zubieta, E. M. (2005). Desarrollo, calidad institucional y valores de la población: El problema de la no inclusión. Alternativas en Psicología, 10(12), 2-10.

Benbenaste, N., Etchezahar, E. \& Del Río, M. (2008). Psicología de la anomia. Anuario de Investigaciones, 15, 187-193.

Beramendi, M. \& Zubieta, E. (2011). La trasgresión como modelado de legitimación. Manuscrito enviado para su publicación.

Blanco, A. \& Díaz, D. (2005). El bienestar social: su concepto y medición. Psicothema, 17(4), 582-589.

Blanco, A. \& Díaz, D. (2007). El rostro bifronte del fatalismo: fatalismo individualista y fatalismo colectivista. Psicothema, 19(4), 552-558.

Carballo, M. (2005). Los argentinos y el mundo del trabajo. Buenos Aires: Nueva Mayoría. 
Delfino, G. I. (2009). Participación politica y factores psicosociales: un estudio con estudiantes universitarios. Tesis doctoral. Facultad de Psicología, Universidad de Buenos Aires.

Durkheim, E. (1897/1976). El suicidio. Madrid: Akal.

Durkheim, E. (1893/1973). De la división del trabajo social. Buenos Aires: Shapire.

Grossi, J. \& Ovejero, A. (1994). Alienación y participación política en la Universidad de Oviedo. Psicología Política, 8, 45-61.

Hegel, G. W. F. (1807/1971). Fenomenología del Espíritu. México: Fondo de Cultura Económica.

Herring, C. (1989). Acquiescence or activism? Political behavior among the politically alienated. Political Psychology, 10(1), 135-153.

Inglehart, R., Basáñez, M., Díez-Medrano, J., Halman, L. \& Luijkx, R. (2004). Human beliefs and values. A cross-cultural sourcebook based on the 1999-2002 values surveys. México: Siglo XXI.

Keyes, C. (1998). Social well-being. Social Psychology Quarterly, 61(2), 121-140.

Laca Arocena, F., Mejía Ceballos, J. C. \& Yañez Velasco, C. (2010). Identidad mexicana e interés político: Predictores de bienestar social y anomia. Acta Universitaria, 20(2), 40-49.

Laca Arocena, F., Santana Aguilar, H., Ochoa Madrigal, Y. \& Mejía Ceballos, J. C. (2011). Percepciones de bienestar social, anomia, interés e impotencia política en relación con las actitudes hacia la democracia. Liberabit, 17(1), 7-18.

Long, S. (1990). Explicando la alienación política. Psicología Política, 1, 87-108.

López Fernández, M. P. (2009). El concepto de anomia de Durkheim y las aportaciones teóricas posteriores. Revista de Ciencias Sociales de la Universidad Iberoamericana, 4(8), 130-147.

Marx, K. (1844/1985). Manuscritos: economia y filosofía. Madrid: Alianza Universidad.

Merton, R. K. (1938). Social structure and anomie. American Psychological Review, 3, 672-682.

Parales-Quenza (2008). Anomia social y salud mental pública. Revista de Salud Pública, 10(4), 658-666. 
Pegoraro, J. S. (2000). Violencia delictiva, inseguridad urbana: la construcción social de la inseguridad ciudadana. Nueva Sociedad, 167, 114-131.

Rodríguez Kauth, A. (2001). Corrupción e impunidad: dos estilos de cultura política latinoamericana. Probidad, 14, 1-12.

Sabucedo, J. M. (1988). Participación política. En J. Seoane \& A. Rodríguez. Psicología politica (pp. 165-194). Madrid: Pirámide.

Seeman, M. (1982). On the meaning of alienation. Current Contents. Social Behavior Science, 37, 22.

Seeman, M. (1991). Alienation and anomie. En J. P. Robinson, P. R. Shaver \& L. S. Wrightsman (Eds.). Measures of Personality and Social Psychological Attitudes (pp. 291-372). San Diego: Academic Press.

Srole, L. (1956). Social integration and certain corollaries: an exploratory study. American Sociological Review, 21(6), 709-716.

Zubieta, E. M. (2008). Valores humanos y conducta social. En M. M. Casullo (Ed.), Prácticas en Psicología Positiva (pp. 203-229). Buenos Aires: Lugar.

Zubieta, E. M., Beramendi, M., Sosa, F. \& Torres, J. A. (2010). Sexismo ambivalente, estereotipos y valores en el ámbito militar. Revista de Psicología, 29(1), 101-130.

Zubieta, E. M. \& Delfino, G. I. (2010). Satisfacción con la vida, bienestar psicológico y bienestar social en estudiantes universitarios de Buenos Aires. Anuario de Investigaciones, 17, 277-283.

Zubieta, E. M., Delfino, G. I. \& Fernández, O. D. (2008). Clima social emocional, confianza en las instituciones y percepción de problemas sociales. Un estudio con estudiantes universitarios urbanos argentinos, Psykhe, 17(1), 5-16.

Zubieta, E., Muratori, M. \& Fernández, O. (2011). Felicidad y Bienestar Psicosocial: ¿una cuestión de género? Manuscrito presentado para su publicación.

Recibido el 23 de noviembre, 2012 Aceptado el 13 de marzo, 2013 